\title{
The neutron cross section of barite-enriched concrete for radioprotection shielding in the range $1 \mathrm{meV}-1 \mathrm{keV}$
}

\author{
Marco Martellucci ${ }^{1}$, Giovanni Romanelli ${ }^{2, a}{ }_{\mathbb{D}}$, Silvio Valeri $^{1}$, Danilo Cottone ${ }^{3}$, \\ Carla Andreani ${ }^{4}$ (i), Roberto Senesi ${ }^{4,5}$ (B) \\ ${ }^{1}$ Mardel Srl, Via Topino 35, Roma, Italy \\ 2 ISIS Facility, Rutherford Appleton Laboratory, Didcot OX11 0QX, United Kingdom \\ 3 Bariblock - SVA S.r.1., Via Meucci 26, 30020 Noventa di Piave, VE, Italy \\ 4 Dipartimento di Fisica and NAST Centre, Università degli Studi di Roma "Tor Vergata”, 00133 Roma, Italy \\ 5 CNR-IPCF, Sezione di Messina, Viale Ferdinando Stagno d'Alcontres 37, Messina 98158, Italy
}

Received: 5 January 2021 / Accepted: 16 February 2021

(C) The Author(s), under exclusive licence to Società Italiana di Fisica and Springer-Verlag GmbH Germany, part of Springer Nature 2021

\begin{abstract}
We present a characterization of a series of barite-enriched concrete samples at the atomic scale using neutron-based techniques. In particular, neutron transmission measurements provide the macroscopic cross section (also known as neutron removal cross section) as a function of the incident neutron energy in the range $1 \mathrm{meV}-1 \mathrm{keV}$. In this range, where fewer experimental investigations are available in the literature, the cross section is dominated by the scattering events from hydrogen, as opposed to the fast-neutron region ( $\mathrm{MeV}$-energy neutrons) where capture events by $\mathrm{Ba}$ are more important. Moreover, below $1 \mathrm{eV}$, the cross section depends on the molecular or crystal structure of the components. For each sample, the amounts of barium and hydrogen are provided by neutron resonance capture analysis and deep inelastic neutron scattering, respectively. We find that the amounts of barium and hydrogen are correlated, with a lower amount of hydrogen in the samples with more barium, likely because of the absence of some calcium-silicon-hydrate structures, whose formation is inhibited by the presence of barite. Moreover, we quantify the non-negligible contribution to the neutron macroscopic cross section arising from water molecules loosely bound in concrete. This contribution makes the shielding performance of concrete dependent upon the humidity and temperature conditions affecting the installation. Our results provide additional guidelines for radioprotection workers to determine the optimal concentration of barite in mixed gamma/neutron radiation environments.
\end{abstract}

\section{Introduction}

Heavy-weight concrete is widely used to protect against $\gamma$-rays and neutrons in medical hospitals, particle accelerators, and nuclear power plants [1-4]. Neutrons have a high penetration power with respect to other radiations, and their scattering and capture cross sections vary dramatically amongst the elements of the periodic table, as well as with the neutron energy. Optimal neutron shielding is provided by elements that can absorb neutrons, yet such events

\footnotetext{
a e-mail: giovanni.romanelli@stfc.ac.uk (corresponding author)
} 
are generally accompanied by the generation of a cascade of secondary $\gamma$-rays, whose energy spectrum and intensity vary from one isotope to another [5,6]. As the attenuation of $\gamma$-rays scales with the atomic number, materials containing heavy-weight elements, such as barites or magnetite, are often used as aggregates in the preparation of heavy-weight concrete for shielding applications.

The concentration of barite $\left(\mathrm{BaSO}_{4}\right)$ in heavy-weight concrete needs to be tuned so as to determine the optimal shielding properties in a mixed $\gamma$-ray and neutron radiation environment. The neutron-attenuation properties for shielding materials are often measured in terms of the removal, or macroscopic, total cross section, $\Sigma_{R}(E)$. This quantity takes into consideration the probability for a neutron of a given energy, $E$, to be removed from an incident beam, either as a consequence of a scattering or capture process. A high concentration of barium provides a large contribution to the capture cross section, $\Sigma_{C}(E)$, corresponding to the absorption of a neutron and the emission of secondary $\gamma$-rays. Moreover, barium provides the means to attenuate both primary and secondary $\gamma$-ray radiation, owing to its high atomic number. However, larger quantities of barite are found to affect the amount of hydrogen in concrete [7]. Hydrogen, while displaying a lower capture cross section than barium, provides the largest contribution to the scattering process. Moreover, scattering from light elements, such as hydrogen, causes a substantial loss of kinetic energy of neutrons, as opposed to the scattering from heavy-weight elements like barium, thus providing moderating capabilities to concrete [7].

Figure 1 reports the calculated [8] capture (left) and total (right) macroscopic cross sections of hydrogen, oxygen, and barium weighted by their typical concentrations in ordinary and baritic concrete. One can readily notice how the capture cross section of barium becomes predominant, for neutrons with energies higher than ca. $1 \mathrm{keV}$, compared to the ones from hydrogen and oxygen. For this reason, a number of experimental investigations on concrete have been performed using $\mathrm{MeV}$ neutrons, e.g. from AmBe radioactive sources $[9,10]$. On the other hand, in the region of thermal $(1 \mathrm{meV}-1 \mathrm{eV})$ and epithermal neutrons $(1 \mathrm{eV}-1$ $\mathrm{keV}$ ), the capture contributions to the cross sections become negligible with respect to the scattering ones. In this region, scattering from hydrogen provides the largest contribution to the macroscopic cross section, despite its relatively little mass percentage with respect to the other elements aforementioned. In fact, up to $54 \%$ of the scattering between $1 \mathrm{eV}$ and $1 \mathrm{keV}$ comes from $\mathrm{H}$.

\section{Materials and methods}

Ordinary and baritic concrete samples were prepared with a thickness of about $1 \mathrm{~cm}$, so as to allow optimal neutron measurements. A barite aggregate commercially available (Sabbia SVA) was used, with an apparent density of $3950 \mathrm{~kg} / \mathrm{m}^{3}$ and a maximum size of the aggregate particles of $0.5 \mathrm{~mm}$. Baritic concretes were obtained using $400 \mathrm{~kg} / \mathrm{m}^{3}$ of cement, $188 \mathrm{l} / \mathrm{m}^{3}$ of water, and $2800 \mathrm{~kg} / \mathrm{m}^{3}$ of Sabbia SVA. Ordinary concrete was obtained using aggregate particles with dimension lower than $16 \mathrm{~mm}$, and mixing $430 \mathrm{~kg} / \mathrm{m}^{3}$ of cement, $160 \mathrm{l} / \mathrm{m}^{3}$ of water, $1200 \mathrm{~kg} / \mathrm{m}^{3}$ of sand, $520 \mathrm{~kg} / \mathrm{m}^{3}$ of gravel, $50 \mathrm{~kg} / \mathrm{m}^{3}$ of fly ashes, and $4.7 \mathrm{l} / \mathrm{m}^{3}$ of Glenium Sky. The measured values of the thickness, $d$, area, $A$, and mass, $M$, of the samples are reported in Table 1.

Neutron experiments were performed at the VESUVIO spectrometer [13] at the ISIS Pulsed Neutron and Muon Source, UK. VESUVIO allows the atomic-scale characterization of a given sample using several techniques at the same time, owing to the wide energy range available at the sample position [11], as evidenced in Fig. 2 (left). Figure 2 (right) provides 

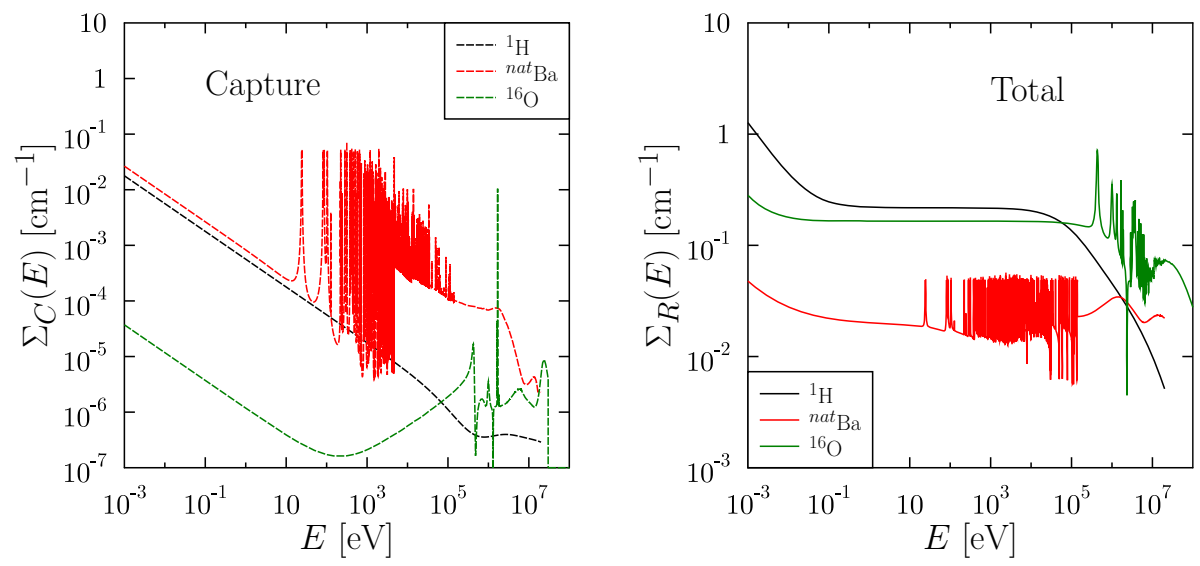

Fig. 1 Capture (left) and total (right) macroscopic neutron cross sections from the ENDF/B-VIII nuclear library [8] in the case of $\mathrm{H}(0.8 \mathrm{wt} \%), \mathrm{O}(52 \mathrm{wt} \%)$, and Ba (49 wt \%). The concentrations of $\mathrm{H}$ and $\mathrm{O}$ are those expected in ordinary concrete $\left(2.4 \mathrm{~g} / \mathrm{cm}^{3}\right)$, while the concentration of barium is that typical of highly enriched baritic concrete [7]

Table 1 Values of the mass $M$, thickness, $d$, area, $A$, and areal density, $\rho_{s}$, for all the samples investigated

\begin{tabular}{llllll}
\hline Sample & $\mathrm{Q}[\mu \mathrm{Ah}]$ & $\mathrm{M}[\mathrm{g}]$ & $\mathrm{d}[\mathrm{cm}]$ & $\mathrm{A}\left[\mathrm{cm}^{2}\right]$ & $\rho_{S}\left[\mathrm{~g} / \mathrm{cm}^{2}\right]$ \\
\hline A & 540 & 58.91 & 1.05 & 25.00 & 2.36 \\
V & 1980 & 60.26 & 1.02 & 24.75 & 2.43 \\
C & 360 & 64.63 & 1.15 & 25.00 & 2.59 \\
F & 1620 & 67.90 & 1.15 & 25.00 & 2.72 \\
T & 177 & 70.08 & 1.10 & 24.88 & 2.82 \\
S & 180 & 71.56 & 1.10 & 25.00 & 2.86 \\
D & 540 & 70.45 & 1.10 & 24.50 & 2.88 \\
R & 360 & 72.55 & 1.15 & 24.63 & 2.95 \\
U & 360 & 73.95 & 1.15 & 24.84 & 2.98 \\
B & 540 & 74.80 & 1.20 & 25.00 & 2.99 \\
\hline
\end{tabular}

The experimental uncertainties on $M, d, A$, and $\rho_{S}$ are constant for all samples and correspond to $0.01 \mathrm{~g}, 0.05$ $\mathrm{cm}, 0.0025 \mathrm{~cm}^{2}$, and $0.001 \mathrm{~g} / \mathrm{cm}^{2}$, respectively. For each sample, the integrated proton current in the ISIS synchrotron, $Q$, is also reported as a measure of the statistics of the neutron data. The uncertainty on the value of $Q$ is on the last digit reported

a schematic representation of the instrument (available at [12]), and different detectors or banks of detectors will be referred to with letters a-d, following the key of the figure. A detailed description of the techniques concurrently employed in the present investigation is provided in the following subsections and, more in general, in Ref. [14].

Prior to the neutron experiments, the samples were wrapped in a $20-\mu \mathrm{m}$-thick Al sachet and attached to an Al circular frame, so that the interaction of neutrons with sample holders could be completely neglected. Samples were measured with the thickness $d$ parallel to the incident neutron beam, at room temperature in vacuum at $10^{-5}$ mbar within the instrument tank, so as to avoid attenuation of the incident neutron beam by air, and to reduce all environmental backgrounds. A slight loss of surface water because of the vacuum conditions has been monitored and is discussed later. For each sample, associated with a capital letter during the 

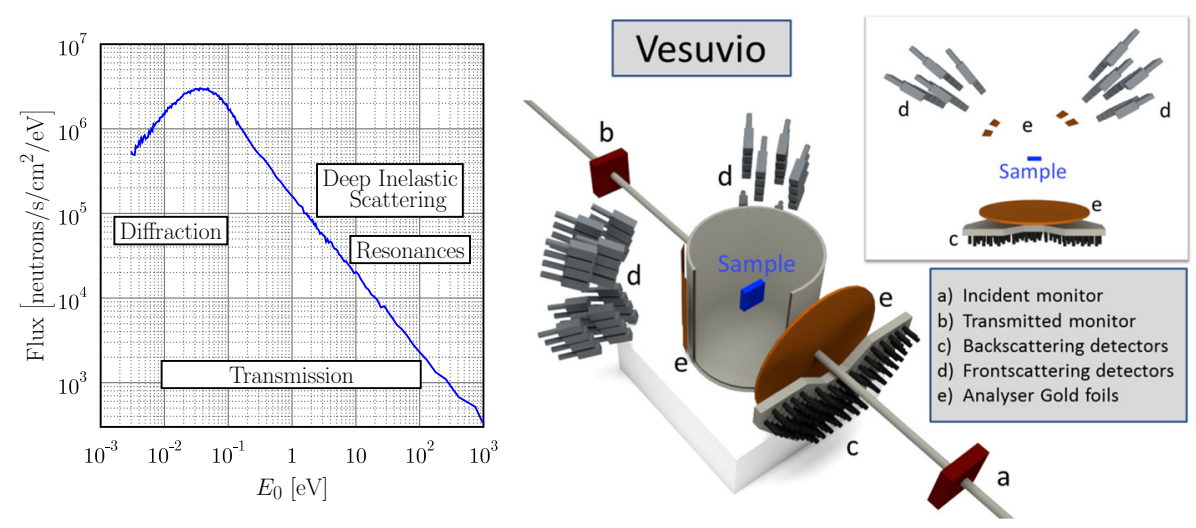

Fig. 2 Neutron flux at the sample position (left, data from ref. [11]), and schematic representation of the VESUVIO spectrometer (right, available at [12])

neutron measurement, the value of the integrated current flown within the ISIS synchrotron during each data acquisition, $Q$, is also reported in Table 1 as an indication of the statistics associated with that measurement.

\subsection{Neutron transmission}

Neutron transmission measurements on VESUVIO [15,16] were performed as a function of the incident neutron energy in the energy range $1 \mathrm{meV}-1 \mathrm{keV}$. Spectra were acquired using the time-of-flight (t.o.f.) technique, available at a pulsed neutron source like ISIS, by using the incident and transmitted ${ }^{6} \mathrm{Li}$-doped scintillator monitors available on the instrument. The data reduction and the conversion from t.o.f. to incident neutron energy, $E$, were handled within the MANTID environment $[17,18]$. The distances of the incident $\left(L_{a}\right)$ and transmitted $\left(L_{b}\right)$ monitors from the room-temperature water moderator facing the VESUVIO spectrometer were calibrated using the dedicated incident foil changer, as discussed in ref. [16]. The conversion from ToF to incident neutron energy is then obtained from the equation (see, for example, ref. [19])

$$
E=\frac{m_{N}}{2} \frac{L_{a, b}^{2}}{(t . o . f .)^{2}},
$$

where $m_{N}$ is the mass of the neutron. Provided that $L$ is expressed in metres, $E$ in eV, and t.o.f. in $\mu$ s, one has that $m_{N}=1.04541 \times 10^{4} \mathrm{eV} \mu \mathrm{s}^{2} / \mathrm{m}^{2}$.

Transmission spectra are interpreted using the Beer-Lambert law, namely [16]

$$
T(E)=\alpha(E) \frac{S_{b}(E)}{C_{b}(E)}=\exp \left(-d \sum_{i} n_{i} \sigma_{i}(E)\right)=\exp \left(-\Sigma_{R}(E) d\right)
$$

where $T(E)$ is the transmission spectrum as a function of the incident neutron energy, $E$, obtained from the ratio of the spectra collected using the transmitted monitor with and without the sample, $S_{b}(E)$ and $C_{b}(E)$, respectively. In order to account for the different duration of the data acquisitions with and without the sample, Eq. 2 is corrected by the parameter $\alpha(E)=C_{a}(E) / S_{a}(E)$, where $S_{a}(E)$ and $C_{a}(E)$ are the spectra measured by the incident monitor with and without the sample, respectively. As the incident spectra are measured before the sample position, they do not depend on the presence of the sample and 
are, therefore, proportional to the incident neutron flux. We notice that $\alpha(E)$ is evaluated as a function of the incident neutron energy, rather than being integrated over all neutron energies, so as to correct for slight changes in the shape of the incident beam flux [20]. Moreover, $n_{i}$ and $\sigma_{i}(E)$ are the number density and total cross section of the crystal or molecular component $i$ within the sample (water, calcium-silicon-hydrate structures, barite, $\mathrm{CaO}, \mathrm{Ca}(\mathrm{OH})_{2}$, etc.). Finally, $\Sigma_{R}(E)$ is the macroscopic, or removal, cross section for a given composition of concrete.

\subsection{Neutron resonance capture analysis}

Neutron resonance capture analysis [14] (NRCA) is based on the nuclear process involving the resonant absorption of a neutron at a given energy, and the emission of a cascade of prompt $\gamma$ rays. VESUVIO is equipped with a set of $\gamma$-sensitive detectors, namely yttrium aluminium perovskite (YAP) scintillators [21,22], that can detect such emitted $\gamma$ rays. The resonance energies are isotope-dependent; thus, NRCA allows the quantitative analysis of some elements and isotopes in a given sample. In the present investigation, NRCA was employed to monitor the amount of barium within the sample by analysing the intensity of its lower-energy nuclear resonances at ca. $100 \mathrm{eV}$. In order to minimize the environmental backgrounds, a lower-level discrimination threshold for the photon energies was set to 600 $\mathrm{keV}$ [23-27]. The relation between incident energy and t.o.f. is (see, for example, ref. [19])

$$
E=\frac{m_{N}}{2} \frac{L_{0}^{2}}{(\text { t.o.f. })^{2}}
$$

where $L_{0}$ is the distance between the sample position and the moderator. The count rate can be expressed as [28]:

$$
C(t . o . f .)=I(E) \alpha(E) \Delta \Omega \sum_{i} N_{i} \eta_{i} \sigma_{\gamma, i}^{D}(E)+B(E),
$$

where $\sigma_{\gamma, i}$ is the capture cross section of isotope $i, N_{i}$ is the number of units of such isotope in the sample, and $B(E)$ is an environmental background. Moreover, $\eta_{i}$ is the efficiency of the YAP detector, considered here to be isotope dependent because only photons with energies higher than the low-level discrimination threshold are detected, and $\Delta \Omega$ the solid angle seen by the detector. Moreover, the attenuation function [28],

$$
\alpha(E)=\frac{1-\exp \left(-\Sigma_{R}(E) d\right)}{\Sigma_{R}(E) d} \simeq \alpha,
$$

is assumed to be constant over all the samples considered in the particular case of the present investigation, as discussed later.

\subsection{Deep inelastic neutron scattering}

Deep inelastic neutron scattering [29,30] (DINS) is an experimental technique based on the regime of the impulse approximation [31-33], whereby the scattering spectra are composed of a series of mass-separated signals referred to as neutron Compton profiles [34]. This is an inelastic scattering in the sense that the incident and final neutron energies are different. In particular, DINS measurements on VESUVIO are performed by selecting the final neutron energy using the resonant capture by ${ }^{197} \mathrm{Au}$ foils placed in front of the forward-scattering detectors (detectors "d" in Fig. 2). In this way, scattered neutrons with energy $E_{R}=4.9$ $\mathrm{eV}$ are converted in prompt $\gamma$ radiation and detected by the same YAP detectors used in the 
NRCA measurements. The incident neutron energy is calculated using the t.o.f. technique, namely [29]

$$
\text { t.o.f. }=\sqrt{\frac{m_{N}}{2}}\left(\frac{L_{0}}{\sqrt{E}}+\frac{L_{d}}{\sqrt{E_{R}}}\right),
$$

where $L_{d}$ is the distance between sample and a given forward-scattering detector. During a DINS measurement, the YAP scintillators also detect a substantial environmental $\gamma$ background. For this reason, an additional gold foil (the component "e" in Fig. 2) is cycled in and out of the trajectory between the sample and the detector, in the so-called foil cycling technique [35]. In this framework, by taking the difference of foil-in and foil-out, the DINS count rate can be expressed as [30]:

$$
C(t)=\frac{E I(E)}{Q} \eta\left(E_{R}\right) \sqrt{\frac{8 E_{R}}{L_{0}^{2} m_{N}}} \Delta \Omega \sum_{i} N_{i} \frac{\sigma_{b, i}^{2}}{4 \pi} M_{i} F\left(y_{i}, Q\right),
$$

where $I(E)$ is the incident flux at the sample position, $Q$ is the neutron momentum transfer, $\eta\left(E_{R}\right)$ is the detector efficiency at the final energy $E_{R}, \Delta \Omega$ is the solid angle as seen by the detector, $M_{i}$ is the nuclear mass of isotope $i, \sigma_{b, i}$ its bound scattering cross section, $N_{i}$ the number of units of isotope $i$ in the sample, $F\left(y_{i}, Q\right)$ is the experimental neutron Compton profile, and $y_{i}$ is the associated West-scaling variable [29,33],

$$
y_{i}=\frac{M_{i}}{\hbar^{2} Q}\left(E-E_{R}-\frac{\hbar^{2} Q^{2}}{2 M_{i}}\right) .
$$

In this experiment, DINS was used to probe the amount of hydrogen in the samples, a capability previously employed, e.g. in refs. [36,37].

\subsection{Neutron diffraction}

Owing to the thermal flux available at the sample position, VESUVIO also allows concurrent neutron diffraction measurements by looking at the elastic scattering of neutrons as measured by the ${ }^{6} \mathrm{Li}$-doped backscattering detectors [11] (detectors "c" in Fig. 2). Here, elastic scattering is defined by the condition that the energies of incident and scattered neutrons are equal. In this case, the t.o.f. can be related to the neutron energy as (see, for example, ref. [19])

$$
E=\frac{m_{N}}{2} \frac{\left(L_{0}+L_{c}\right)^{2}}{(\text { t.o.f. })^{2}},
$$

where $L_{c}$ is the distance between sample and backscattering detectors. In this experiment, neutron diffraction was used to monitor the changes in the structure at the microscopic scale as a function of the content of barite in the samples.

\section{Results and discussion}

Figure 3 (left) shows the experimental macroscopic cross section of three samples with different barite content. The presence of barium in samples $C$ and $D$, is clearly manifested in the isotope-specific nuclear resonances in the energy region above ca. $10 \mathrm{eV}$. One can notice how, for higher concentrations of barium, the overall macroscopic cross section decreases over the entire energy range investigated. This is clearly related to a lower amount of hydrogen (either structural or from water) in the sample, as discussed later. The difference between spectra A 

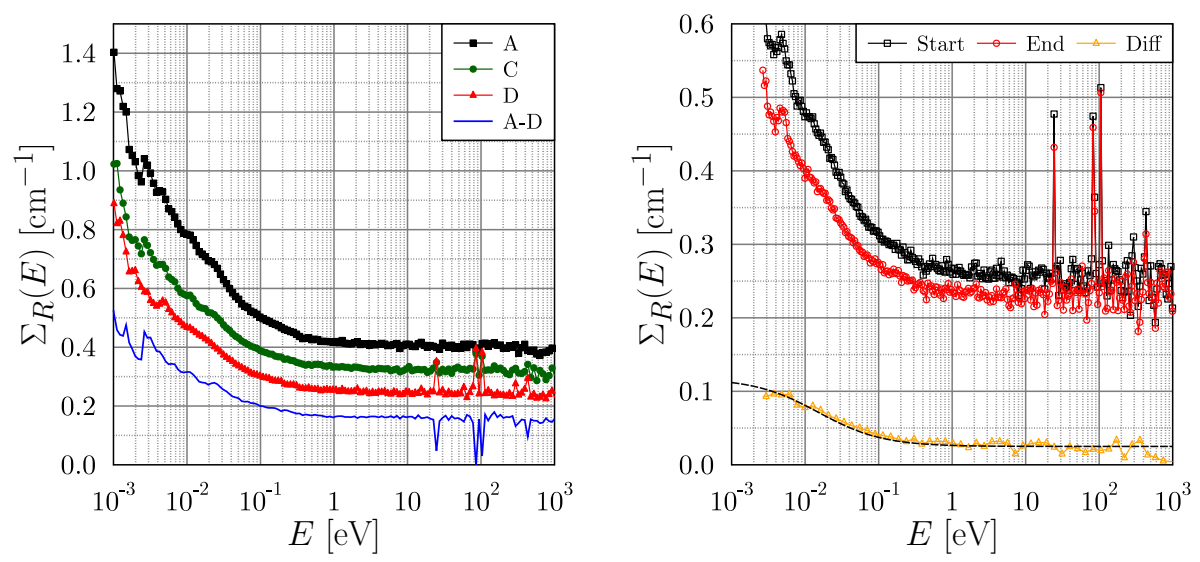

Fig. 3 Left: macroscopic, or removal, total cross section, $\Sigma_{R}(E)$ of cements with different amount of barite as a function of the incident neutron energy, $E$. The difference of spectra A and D is reported as a blue line. Right: macroscopic cross section of sample F at the start (black squares) and at the end (red circles) of a 12-hour measurement under vacuum. Their difference is reported as orange triangles, and it is compared to the model from Ref. [40]

and $\mathrm{D}$ is reported as a blue line and is the result of the combination of a decreasing contribution from the hydrogen content and an increasing contribution from other components of the concrete, as barite. For this reason, the A-D difference has a markedly different shape than the cross section of hydrogen in water [38,39] or other organic samples [40-43]. Considering the energy dependence of the hydrogen cross section (Fig. 1), the results for sample A, with no barite, are compatible with those reported in ref. [44], where the calculated values for the hydrogen removal cross section for fast neutrons were ca. $1.5 \times 10^{-2} \mathrm{~cm}^{-1}$.

Figure 3 (right) shows the change in the macroscopic cross section for sample $\mathrm{F}$ after a period of 12 hours in vacuum. The reduction in $\Sigma_{R}(E)$ is clearly related to the loss of some loosely bound water in the sample. The difference of the measured spectra at the start (black squares) and at the end (red circles) of the 12-hour period is reported as orange triangles in the same figure. This difference has the same energy dependence as other hydrogen total cross sections in organic samples, as one can appreciate by the comparison with the model presented in ref. [40], reported as black dashed line in the same figure, confirming that it is related to the water loss. It is also important to note that in the region between hundreds of $\mathrm{meV}$ and few $\mathrm{eV}$, the shape of the total cross section, in the short time collision approximation $[43,45]$, mainly depends on the effective temperature of hydrogen in the system, thus on its nuclear kinetic energy, that is generally much higher than the thermodynamic one still at room temperature [46-50].

The amounts of barium and hydrogen in each sample were monitored, concurrently to the neutron transmission experiment, using NRCA and DINS, respectively. The NRCA and DINS spectra from samples A, C, and D are reported in the left and right panels of Fig. 4, respectively.

In the case of NRCA spectra, reported in Fig. 4 (left), a series of sharp and intense peaks in the t.o.f. spectra in the region $60-180 \mu$ s correspond to the resonant capture by ${ }^{135} \mathrm{Ba}$ of epithermal neutrons at energies of 82,87, and $104 \mathrm{eV}$, resulting in a cascade of $\gamma$ rays detected by the YAP detectors. The figure shows how no barium is present in sample A (black squares), while the amount of barium increases for the higher density samples $\mathrm{C}$ and $\mathrm{D}$. As mentioned, 

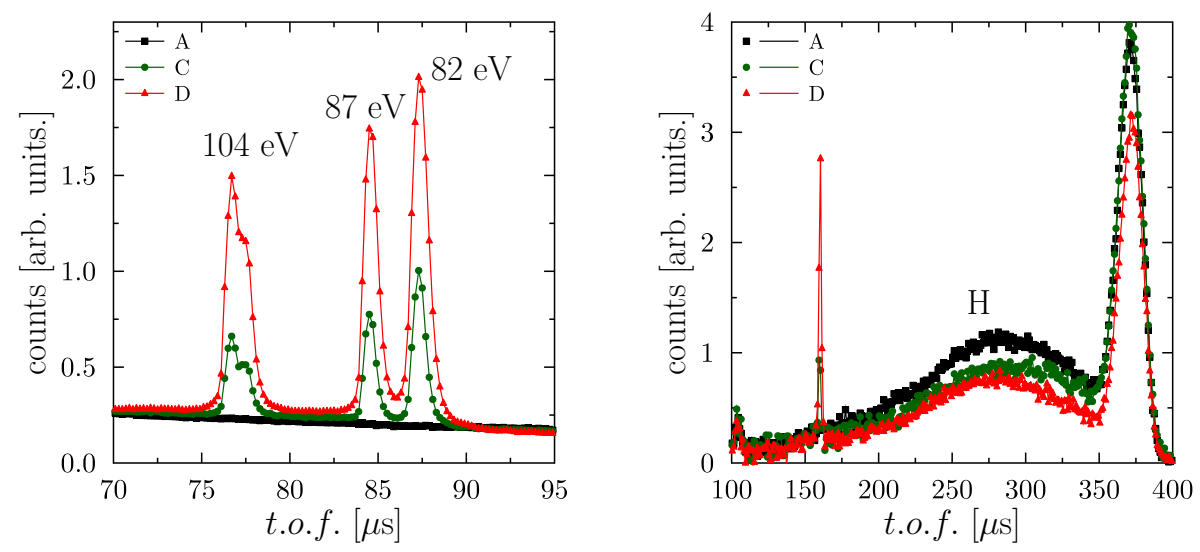

Fig. 4 NRCA (left) and DINS (right) t.o.f. spectra of several samples. All intensities are reported in arbitrary units. A qualitative comparison between the NRCA and DINS intensities is possible by multiplying the NRCA spectra on the left by a factor ca. $10^{3}$

these spectra were not corrected by self-attenuation, according to Eq. 4. The assumption that the self-attenuation coefficient is not sample dependent can be explained, a posteriori, by noticing that $\Sigma_{R}(E)$ (left panel in Fig. 3) is dominated by the constant scattering cross section with respect to the capture one, making it safe to assume $\alpha(E) \simeq \alpha$. Moreover, the self-attenuation correction is not relevant for the determination of the amount of $\mathrm{Ba}$ as different samples, around the values of the mentioned resonances, have similar removal cross sections.

DINS spectra of samples A, C, and D, are reported in Fig. 4 (right). The hydrogen neutron Compton profile is the most intense of the peaks, in the t.o.f. region around 200-300 $\mu$ s. The peak at lower t.o.f. values, ca. $160 \mu$ s, is a residual background from a low-lying resonance of barium, not completely cancelled out after the application of the foil cycling technique. The third peak at ca. 350-400 $\mu \mathrm{s}$, is the result of the superposition of the neutron Compton profiles from all elements heavier than hydrogen, including oxygen, calcium, and barium. One should note that a better mass resolution in a DINS experiment, e.g. to separate the oxygen peak from the heavier elements, is available by using the backscattering detectors (refer to refs. [51-53]).

The previous results clearly suggest that to higher concentrations of barite correspond lower concentrations of hydrogen. This feature was already discussed in ref. [7], where the hydrogen fraction by weight was reported to drop from $0.83 \%$ (for a sample with no barite) to $0.44 \%$ (for a sample with maximum barite content of ca. $83 \mathrm{wt} \%$ ), corresponding to a decrease by a factor of ca. 0.53. Figure 5 (left) shows, for each sample reported in Table 1, the relative amounts of barium and hydrogen as obtained by NRCA and DINS, respectively. These points are divided in two groups, as further discussed in the next paragraph: those with saturated amount of barium, with $N_{B a} / \rho_{s} \simeq 1$, reported as red triangles, and those with lower values of $N_{B a} / \rho_{s}$, reported as green circles. Only samples A, C, and D are explicitly labelled, for the sake of clarity. All samples with higher density reported in Table 1 show a saturated amount of barium, as well as a drop of the hydrogen signal by a factor $0.5-0.6$, well in agreement with ref. [7].

The results from the concurrent diffraction measurements, shown in Fig. 5 (right), clearly show the presence of barite in the denser samples (red spectra), as one can appreciate by 

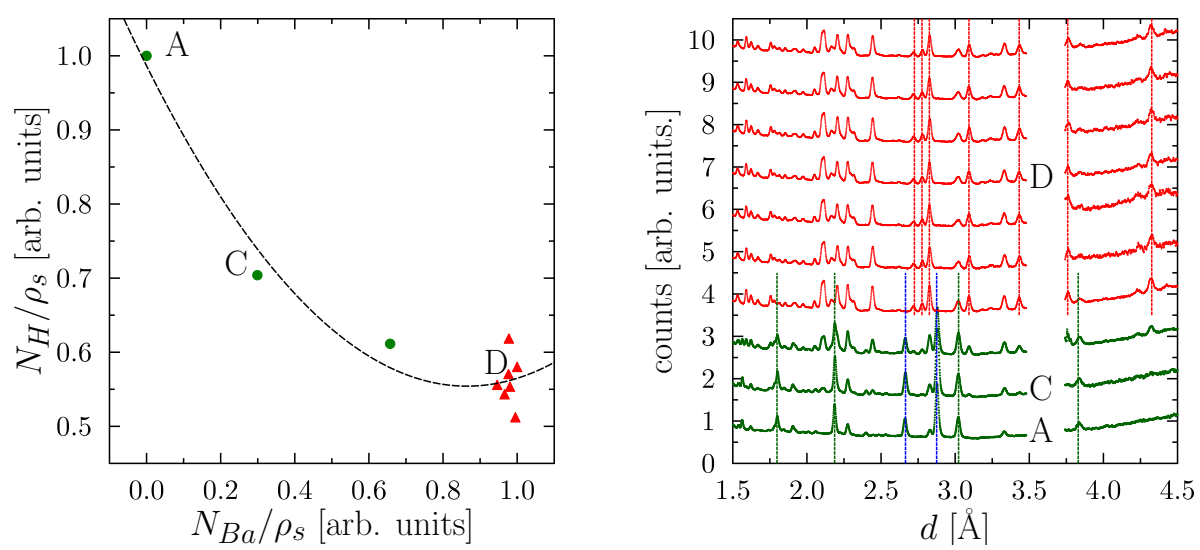

Fig. 5 Left: correlation of the hydrogen and barium amounts in each sample investigated, as obtained from DINS and NRCA, respectively. Right: neutron diffraction spectra of all samples investigated. In both panels, data from samples from Table 1 with lower and higher amount of barium are reported in green and red, respectively. For the sake of clarity, only samples A, C, and D are explicitly labelled

comparing to the reflections from ref. [54] (red vertical dotted lines) at higher $d$-spacing values. Moreover, as the amount of barite increases, some peaks are attenuated, and the peaks at $1.798,2.187,2.665$, and $2.875 \AA$ completely disappear. It has been noted $[55,56]$ that as a consequence of the increasing amount of $\mathrm{BaO}$ in concrete, there is a depletion of alite in the sample. The decrease in the peaks at ca. 3.83, 3.02, 2.19, and $1.80 \AA$ is likely related to the decrease in afwillite [57], possibly resulting from the hydration of alite in concrete, consistently with the results in refs. [55,56]. Moreover, the peak at ca. $2.65 \AA$ could be related to the one in $\mathrm{Ca}(\mathrm{OH})_{2}$ (2.62 in ref. [58]). The decision to divide, in Fig. 5 (right), the diffraction patterns in red and green curves follows the distinction made in the left panel of the same figure. In fact, the denser samples in table 1 are those showing a saturated amount of barium (red triangles in the left panel) as well as the clear presence of the Bragg peaks from barite (red patterns in the right panel). The patterns from samples A, C, and D are explicitly labelled.

It is important to note that in ref. [7], hydrogen was all associated with the water content in the sample, and the decrease in hydrogen was considered tantamount to the decrease in water. However, our results suggest that the hydrogen loss is also related to structural hydrogen from calcium-silicon-hydrate structures formed during the setting of the concrete samples. It is also important to note that the shape of the scattering cross section below $1 \mathrm{eV}$ markedly depends on the chemical environment affecting hydrogen, as previously discussed in Fig 3. Therefore, associating the hydrogen loss solely with a water loss would correspond to a miscalculation of the cross section for thermal neutrons.

\section{Conclusions}

We have presented new measurements of the macroscopic cross section of a series of bariteenriched concrete samples in the neutron energy range $1 \mathrm{meV}-1 \mathrm{keV}$. We found that the cross section is dominated by the scattering contribution off hydrogen, and that the amount of hydrogen decreased as the concentration of barite increased. We also related the decrease 
in the hydrogen level to the lack of some crystal structures, whose formation upon the setting of the sample can be inhibited by the presence of barite or barium oxide.

Moreover, we showed that a non-negligible contribution to the macroscopic cross section arises from water molecules loosely bound in concrete, providing differences of up to $10 \%$ when the sample is pumped in vacuum. This results showed how the shielding properties of concrete can be affected by the temperature and humidity conditions affecting a given installation.

Acknowledgements The authors gratefully acknowledge the financial support of Regione Lazio (IR approved by Giunta Regionale n. G10795, 7 August 2019 published by BURL n. 6927 August 2019), ISIS@ MACH (I), and ISIS Neutron and Muon Source (UK) of Science and Technology Facilities Council (STFC); the financial support of Consiglio Nazionale delle Ricerche within CNR-STFC Agreement 2014-2020 (N 3420), concerning collaboration in scientific research at the ISIS Neutron and Muon Source (UK) of Science and Technology Facilities Council (STFC), is gratefully acknowledged. We thank the STFC Rutherford Appleton Laboratory for access to neutron beam facilities (VESUVIO, https://doi.org/10.5286/ISIS.E.RB2000164).

Data Availability Statement This manuscript has associated data in a data repository. [Authors' comment: Raw data related to this article were generated at the ISIS Neutron and Muon Source (UK), DOI: https:// doi.org/10.5286/ISIS.E.RB2000164. Derived data supporting the findings of this study are available from the corresponding author upon reasonable request.]

\section{References}

1. K. Sakr, W. Ramadan, M. Sayed, T. El-Zakla, M. El-Desouqy, N. El-Faramawy, Radiat. Eff. Defects Solids 173(3-4), 269-282 (2018)

2. T. Piotrowski, D. Tefelski, A. Polański, J. Skubalski, Open Eng. 2(2), 296-303 (2012)

3. B. Oto, A. Gur, E. Kavaz, T. Cakir, N. Yaltay, Prog. Nucl. En. 92, 71-80 (2016)

4. A. Mesbahi, H. Ghiasi, App. Rad. Isot. 136, 27-31 (2018)

5. G. Molnar, Handbook of Prompt Gamma Activation Analysis: With Neutron Beams, vol. 1 (Springer Science \& Business Media, Berlin, 2004)

6. T. Belgya, Z. Kis, L. Szentmiklósi, Z. Kasztovszky, P. Kudejova, R. Schulze, T. Materna, G. Festa, P.A. Caroppi, J. Rad. Nucl. Chem. 278(3), 751-754 (2008)

7. I. Akkurt, A.M. El-Khayatt, Ann. Nucl. En. 51, 5-9 (2013)

8. D. A. Brown, M. B. Chadwick, R. Capote, A. C. Kahler, A. Trkov, M. W. Herman, A. A. Sonzogni, Y. Danon, A. D. Carlson, and others. Nucl Data Sheets, 148:1 - 142, (2018). Special Issue on Nuclear Reaction Data

9. E. Gallego, A. Lorente, H.R. Vega-Carrillo, Nucl. Tech. 168(2), 399-404 (2009)

10. R. Khelifi, Z. Idiri, L. Omari, M. Seghir, App. Rad. Isot. 51(1), 9-13 (1999)

11. G. Romanelli, M. Krzystyniak, R. Senesi, D. Raspino, J. Boxall, D. Pooley, S. Moorby, E. Schooneveld, N.J. Rhodes, C. Andreani, F. Fernandez-Alonso, Meas. Sci. Tech. 28(9), 095501 (2017)

12. http://www.isis.stfc.ac.uk/Pages/VESUVIO.aspx, Last accessed on February (2021)

13. J. Mayers, G. Reiter, Meas. Sci. Tech. 23, 045902 (2012)

14. M. Krzystyniak, G. Romanelli, M. Fabian, M. Gutmann, G. Festa, L. Arcidiacono, M. Gigg, K. Drużbicki, C. Andreani, R. Senesi, F. Fernandez-Alonso, J. Phys.: Conf. Ser. 1021(1), 012026 (2018)

15. G. Romanelli, S. Rudić, M. Zanetti, C. Andreani, F. Fernandez-Alonso, G. Gorini, M. Krzystyniak, G. Škoro, Nucl. Inst. Meth. A 888, 88-95 (2018)

16. J.I. Robledo, J. Dawidowski, J.I. Márquez Damián, G. Škoro, C. Bovo, G. Romanelli, Nucl. Inst. Meth. A 969, 164096 (2020)

17. http://download.mantidproject.org/, Last accessed on February (2021)

18. G. Romanelli, B. Hewer, M. Krzystyniak, M. Gigg, R. Tolchenov, S. Mukhopadhyay, F. FernandezAlonso, J. Phys.: Conf. Ser. 1055, 012016 (2018)

19. F. Fernandez-Alonso, D.L. Price (eds.), Neutron Scattering - Fundamentals (Academic Press, New York, 2013)

20. G. Skoro, R. Bewley, S. Lilley, R. Ewings, G. Romanelli, M. Gutmann, R. Smith, S. Rudic, S. Ansell, J. Phys.: Conf. Ser. 1021, 012039 (2018)

21. C. Andreani, A. Pietropaolo, R. Senesi, G. Gorini, M. Tardocchi, A. Bracco, N. Rhodes, E. Schooneveld, Nucl. Inst. Meth. A 481, 509-520 (2002) 
22. A. Pietropaolo, C. Andreani, A. D’Angelo, R. Senesi, G. Gorini, S. Imberti, M. Tardocchi, N. Rhodes, E.S. Schooneveld, App. Phys. A: Mater. Sci. Process. 74, 189-190 (2002)

23. M. Tardocchi, A. Pietropaolo, C. Andreani, A. Bracco, A. D’Angelo, G. Gorini, S. Imberti, R. Senesi, N.J. Rhodes, E.M. Schooneveld, Nucl. Inst. Meth. A 526, 477-492 (2004)

24. C. Andreani, A. D’Angelo, G. Gorini, S. Imberti, A. Pietropaolo, N.J. Rhodes, E.M. Schooneveld, R. Senesi, M. Tardocchi, App. Phys. A: Mater. Sci. Process. 78, 903-913 (2004)

25. P. Ulpiani, G. Romanelli, D. Onorati, A. Parmentier, G. Festa, E. Schooneveld, C. Cazzaniga, L. Arcidiacono, C. Andreani, R. Senesi, Rev. Sci. Inst. 90(7), 073901 (2019)

26. D. Onorati, G. Romanelli, P. Ulpiani, C. Cazzaniga, E. Preziosi, L. Arcidiacono, G. Festa, C. Andreani, R. Senesi, M.C. Morone, Nucl. Inst. Meth. A 969, 164012 (2020)

27. A. Pietropaolo, C. Andreani, M. Rebai, L. Giacomelli, G. Gorini, E.P. Cippo, M. Tardocchi, A. Fazzi, G.V. Rinati, C. Verona, M. Marinelli, E. Milani, C.D. Frost, E.M. Schooneveld, Europhys. Lett. 94, 62001 (2011)

28. H. Postma, P. Schillebeeckx, in Encyclopedia of Analytical Chemistry, eds. by R.A. Meyers, R.A. Meyers (2009). https://doi.org/10.1002/9780470027318.a9070

29. C. Andreani, R. Senesi, M. Krzystyniak, G. Romanelli, and F. Fernandez-Alonso. Chapter 7 - Atomic quantum dynamics in materials research. In Felix Fernandez-Alonso and David L. Price, editors, Neutron Scattering - Applications in Biology, Chemistry, and Materials Science, volume 49 of Experimental Methods in the Physical Sciences, pages 403 - 457. Academic Press, (2017)

30. C. Andreani, M. Krzystyniak, G. Romanelli, R. Senesi, F. Fernandez-Alonso, Adv. Phys. 66(1), 1-73 (2017)

31. J.M.F. Gunn, M. Warner, Z. Phys. B Condens. Matter 56(1), 13-20 (1984)

32. V.F. Sears, Phys. Rev. B 30, 44-51 (1984)

33. G.B. West, Phys. Rep. 18, 263-323 (1975)

34. C. Andreani, D. Colognesi, J. Mayers, G.F. Reiter, R. Senesi, Adv. Phys. 54(5), 377-469 (2005)

35. E.M. Schooneveld, J. Mayers, N.J. Rhodes, A. Pietropaolo, C. Andreani, R. Senesi, G. Gorini, E. PerelliCippo, M. Tardocchi, Rev. Sci. Inst. 77, 5103 (2006)

36. M. Krzystyniak, G. Romanelli, F. Fernandez-Alonso, Analyst 144, 3936-3941 (2019)

37. G. Romanelli, A. Liscio, R. Senesi, R. Zamboni, E. Treossi, F. Liscio, G. Giambastiani, V. Palermo, F. Fernandez-Alonso, C. Andreani, Carbon 108, 199-203 (2016)

38. C. Andreani, R. Senesi, M. Krzystyniak, G. Romanelli, F. Fernandez-Alonso, Riv. Nuovo Cimento 41, 291-340 (2018)

39. J.I.M. Damian, J. Dawidowski, J.R. Granada, F. Cantargi, G. Romanelli, C. Helman, M. Krzystyniak, G. Skoro, D. Roubtsov, EPJ Web Conf. 239, 14001 (2020)

40. S.C. Capelli, G. Romanelli, J. Appl. Crystallogr. 52(5), 1233-1237 (2019)

41. F. Cantargi, J. Dawidowski, C. Helman, J.I.M. Damian, J.R. Granada, G. Romanelli, J.G. Cuello, G. Skoro, M. Krzystyniak, EPJ Web Conf. 239, 14002 (2020)

42. L.A.R. Palomino, J. Dawidowski, J.I.M. Damián, G.J. Cuello, G. Romanelli, M. Krzystyniak, Nucl. Inst. Meth. A 870, 84-89 (2017)

43. J. Dawidowski, L.A.R. Palomino, G. Romanelli, G.J. Cuello, J.I.M. Damián, J.I. Robledo, M. Krzystyniak, Nucl. Inst. Meth. A 989, 164948 (2021)

44. E. Yilmaz, H. Baltas, E. Kiris, İ. Ustabas, U. Cevik, A.M. El-Khayatt, Ann. Nucl. En. 38(10), 2204-2212 (2011)

45. D. E. Parks, J. R. Beyster, M. S. Nelkin, N. F. Wikner, Slow Neutron Scattering and Thermalization with Reactor Applications (W. A. Benjamin Inc, New York, 1970)

46. C. Andreani, G. Romanelli, R. Senesi, J. Phys. Chem. Lett. 7(12), 2216-2220 (2016)

47. R. Senesi, G. Romanelli, M.A. Adams, C. Andreani, Chem. Phys. 427, 111-116 (2013)

48. R. Senesi, D. Flammini, A.I. Kolesnikov, E.D. Murray, G. Galli, C. Andreani, J. Chem. Phys. 139(7), 074504 (2013)

49. G. Romanelli, R. Senesi, X. Zhang, K.P. Loh, C. Andreani, Probing the effects of $2 \mathrm{~d}$ confinement on hydrogen dynamics in water and ice adsorbed in graphene oxide sponges. Phys. Chem. Chem. Phys. 17(47), 31680-31684 (2015)

50. C. Andreani, G. Romanelli, A. Parmentier, R. Senesi, A.I. Kolesnikov, H.Y. Ko, M.F.C. Andrade, R. Car, J. Phys. Chem. Lett. 11(21), 9461-9467 (2020)

51. G. Romanelli, M. Ceriotti, D.E. Manolopoulos, C. Pantalei, R. Senesi, C. Andreani, J. Phys. Chem. Lett. 4(19), 3251-3256 (2013)

52. A. Perrichon, E.J. Granhed, G. Romanelli, A. Piovano, A. Lindman, P. Hyldgaard, G. Wahnstrom, M. Karlsson, Chem. Mat. 32(7), 2824-2835 (2020)

53. G. Romanelli, F. Fernandez-Alonso, C. Andreani, J. Phys.: Conf. Ser. 571, 012003 (2014)

54. H. Sawada, Y. Takéuchi, Z. Krist. - Cryst. Mater. 191(3-4), 161-171 (1990) 
55. N.K. Katyal, S.C. Ahluwalia, R. Parkash, Cem. Concr. Res. 29(11), 1857-1862 (1999)

56. A. Zezulova, T. Stanek, T. Opravil, Proc. Eng. 151, 42-49 (2016)

57. H.D. Megaw, Acta Crys. 5(4), 477-491 (1952)

58. W.R. Busing, H.A. Levy, J. Chem. Phys. 26(3), 563-568 (1957) 